\title{
Triad model: simulation - functional tensometry - information database in the assessment of the reliability of technological machines
}

\author{
Nadezhda Sevryugina ${ }^{1, *}$ and Pavel Kapyrin ${ }^{2}$ \\ ${ }^{1}$ Russian State Agrarian University - Moscow Timiryazev Agricultural Academy, Timiryazevskaya \\ St., 49, Moscow, 127550, Russia \\ ${ }^{2}$ Moscow State University of Civil Engineering, Yaroslavskoe shosse, 26, Moscow, 129337, Russia
}

\begin{abstract}
The concept of a multidisciplinary approach to assessing the resource of individual components of the machine by combining database information based on simulation techniques and functional tensomethration is proposed. Simulations determine the reperative points of the tendometric sensors. The creation of a diagnostic model using basic concepts of information theory has allowed the development of a synergistic model for the recognition of the area of displacement of areas of uncertainty, which will ensure the identification of the defect (risk-denial). The formation of an electronic database of parametric data on the nature of loads as a diagnostic indicator of the change in the accuracy of pairing in machine systems is justified. Experimental studies were conducted on the model of the quick-capler. Hierarchical structuring of the machine to the level of mating parts with digital control of the criticality of the magnitude of external and internal loads ensures reliability control throughout the entire service life of the machine. When disposing of machines, this data allows you to obtain information about the residual resources of the elements for their reuse or the feasibility of restoration. This, in turn, will ensure the environmental friendliness and economy of the process.
\end{abstract}

\section{Introduction}

The representation of a technical system as interconnected parameters is one of the methods to establish not only the degree of influence, but also to determine the technical condition by of diagnostic recognition of the intensity of parameter changes.

As it is known, the unit is a complex technical system characterized by geometric, structural and functional parameters, the values of which were determined in the preoperational period, after commissioning, go into the category of partially determined in the presence of a built-in control and measuring apparatus for assessing the state or uncertain indicators, in case of their absence [1-5].

\footnotetext{
* Corresponding author: nssevr@yandex.ru
} 
Uncertainty of the state of a technical system occurs in the following cases: lack of information on the reliability of equipment (high error values); human factor (operator errors), etc.

Scientific developments of the solution to the indicated problem can be represented in the following areas:

- functional efficiency of technical systems:

A.P. Kuznetsov et al [6], conducted research in the field of resource conservation, developed an energy information model with a basic criterion - the efficiency of the functioning of a mechanical system. Also, results were obtained on the development of a mechanism for managing technological change, carried out by Hsin-HuiChou and JudyZolkiewski [7].

Niko Siltala, et al. [10]. substantiate that an integral part of resource assessment is the concept of executable capabilities, which describes the resource management interface in a vendor-neutral form [8].

S.I. Chalaganidze et al. [9] showed the importance of reliability for agricultural machinery that operate in harsh soil-climatic and dynamic conditions, in contrast to some other industrial machinery and equipment.

- theoretical solution by developing mathematical models $[10,11]$ :

Valerie C.Y. Zhu et al. [10]. presented a model of the change in the quality of the system as a function proportional to a linear function of time through a polynomial solution to minimize the total resource consumption within the given limiting limits.

- innovations [12-13].

Xiangxiang Sun et al. [12] assess the impact of market segmentation on the practical implementation of technological innovations, especially in small towns and rural settlements with a low technical standard of living.

Hung-ChunHuang et al. [13], solving a similar problem, argue that technological embeddedness incubates innovation potential and propose to pay special attention to brokerage technological positions, bringing them out as an innovative pillar for interdisciplinary innovation.

In order to reduce innovation risks, in the work of Delu Wang et al. [14] it is proposed to combine the coefficient of variation method, system clustering and combine multiclassifiers for early risk warning with 20 indicators in three dimensions with a decrease in the indicator. The significance of the system-forming property of a selforganizing intelligent data analyzer is substantiated in comparison with traditional singleclassification models (logistic regression, machine-like systems, neural network, decision tree) and six commonly used methods of merging multiclassifiers (such as expert judgment, Bayesian method and genetic algorithm).

Considering the system as a combination of elements structured to perform a separate function, when solving problems of performance assessment, the variability of the state is taken, which in the model is characterized both quantitatively and qualitatively by comparing with the normalized values of the parameters [15].

The purpose of the analysis of the uncertainty of the state of a technical system is to translate the uncertainty of the initial parameters and assumptions used when the risk assessment into the uncertainty of the results. To achieve this goal, it is necessary to solve a number of systemic problems, among which the key ones are the problems of searching for the identification of sources of uncertainty, modal analysis of the information content of the results and their presentation in the algorithmic form of digital recognition for the subsequent creation of a mechanism for managing the risk of failures of a technical system. 


\section{Materials and methods}

\subsection{Modal analysis of technical systems}

"The internal field will be characterized by the design, i.e. the basic condition of achieving the target function, with the laid extreme of existence: maximum and minimum, and formed in the design -production period of the life cycle of the machine. The outer field is formed during the period of operation and is probabilistic, which allows the physical essence of the processes to present in accordance with the variation principle, the interpretation of which is that of all possible move ments nature chooses the one at which the purpose of movement is achieved at the lowest cost of action. As you know, the speed attainment of the limit state of pairing, in particular, in wear, depends on the friction that occurs when touching the matched surfaces, and friction is caused by movement, which in turn arises from the condition of having one or more degrees of freedom in the pairing" [1].

Modern tensomether sensors located in the reparation points of the pairing provide the collection of information and analytical data on the indicator of tribyometry (from Greek tribos - Friction and metreo - Measure). Installation of tensometry sensors and the use of a software machine collecting information ensures the digitization of technical systems at the level of elemental pairing of parts [1-2].

The estimated value of resource potential is formed by the value of the period of intensity of the change in the parameter $\xi(t)$ to the level of reaching its limit value $\xi\left(t_{\lim }\right)$ :

$$
t_{b a l}=t\left[\left(\frac{\xi\left(t_{\lim }\right)}{\xi(t)}\right)^{\frac{1}{\alpha}}-1\right]
$$

where is $\alpha$-the degree to which the nature of the change in the parameter.

\subsection{Simulation model of loading matching elements of the machine's working body}

The task of the designer is to create a working equipment or working body that would meet the requirements of strength and rigidity, were reliable in operation and durable, had less metal intensity and better technical parameters.

In the first stage of the design determine the basic configuration of the element, the ways of its attachment to the nodes of the machine, assign in the first approximation preliminary sizes and shape of the cross-section, depending on the working conditions of the element in the machine, external loading, technology and labor-intensive manufacture. In the second stage of design, the strained state of the element is assessed, its strength indicators are determined and the shape and size are adjusted.

The simulation model is presented by the example of changes in the dynamics of the attachment axes of the kvik-capler at loads caused by working equipment, in particular the installation of buckets of different volumes (fig.1). 


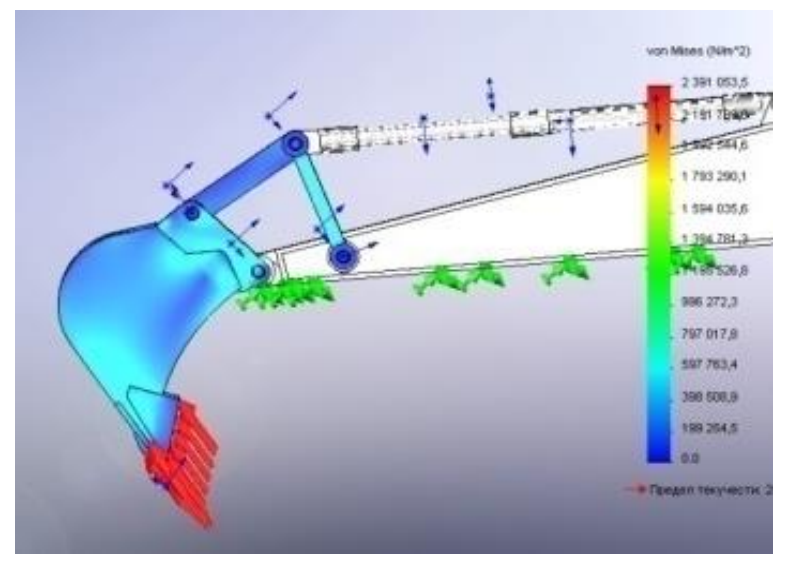

Fig. 1. Simulationof the loading mode when the excavator performs the digging process.

Simulation enables, with minimal material and manpower, to conduct research to assess the effectiveness of the developed design. For the operation of the machines, it becomes important to extend the resource of the entire machine, which is provided by service impacts. In simulation, places to install sensors, reparation points were identified. The collection of load data allows you to determine the degree of deviation of pairing parameters from limited, which allows corrective service impacts. Further studies have been carried out to establish the consistency of the proposed theory and experimental results. Obtaining information about the locations of the reparation points will additionally create a data bank on the amount of external and internal loads in all connections of the machine's systems and monitor its reliability throughout the life of the machine. When disposed of, this information will provide information about the residual resources of the elements for their reuse or the purposefulness of recovery, which in turn will ensure environmental friendliness and cost-effectiveness for machines owners $[1,5]$.

\section{Results and discussions}

Thedefinition of a tensely deformed state in the "the hilt- kvik-capler - bucket" system used a measuring system, whichincludes: sensitive element - tensoresiist; measuring module for dynamic measurements; PC data transmission module; smart sensor cable connection to the measuring network; software.

The structurally measuring system includes an intelligent sensor, which consists of a primary converter (tenzomost) and a measuring module ZET 7111 Tensometer-CAN [23].

The tenzomost is installed at the measurement site, the measuring module is in close proximity. At the exit of the tensomost, a voltage proportional to the impact of the measured value is formed. The measuring module converts this voltage into the values of the measured value and transmits the results digitally. Software was used to display and process measurement results ZETLab. Measurements do not require setting up a sensor and processing the results - all the necessary settings are set by the manufacturer when the smart sensor is released and stored in the memory of the module ZET 7111 and all measurements are made by a signal processor that is part of the measuring module ZET 7111 TensometerCAN. Intelligent tensaters ZET 7111 Tensometer-CAN with basic settings measure the values of relative deformation. Distributed measuring networks are built on the basis of intelligent sensors. To connect the measuring network to systems ZETLAB и ZETVIEW is used interface converter ZET 7174 [2]. 
A model with elements modeling the handle and a working organ has been created to carry out tenzometric measurements. The connectors of the structure used a two-way solid seam of the brand's joint without a slant and edges (for each wall) rolled joint $6-8 \mathrm{~mm}$.

The next step was to prepare the places of fixing tensorestors: stripping areas with sandpaper and skimming.

The tensoressers used were installed on the surface next to the front pairs of the lower and upper sidewall bracket.

The resulting design is installed in a hydraulic press and is created to increase the voltage in the structure to establish the moment of deformation with ever-increasing effort.

With the help of the collected measuring network, tests of excavator working equipment were also carried out to determine the tense-deformed condition in the metal structures of the real machine at the joints of the handle and the working body.

Experimental studies have found that the technological use of the meter system discussed above is not possible due to the low sensitivity of tensorestors, and therefore it was decided to use force as a measuring sensor. Tensometry was carried out on the example of the study of the tense-deformed state of the working organ of the technologicalmachine, in the case of the excavator bucket using a kvik-a droper (fig2).
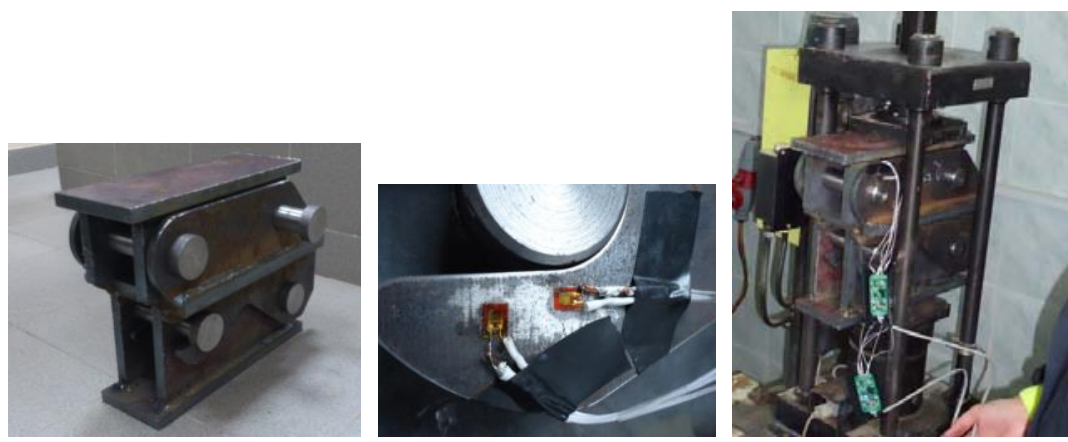

a) installation of tensaters on the model of the kvik-capler
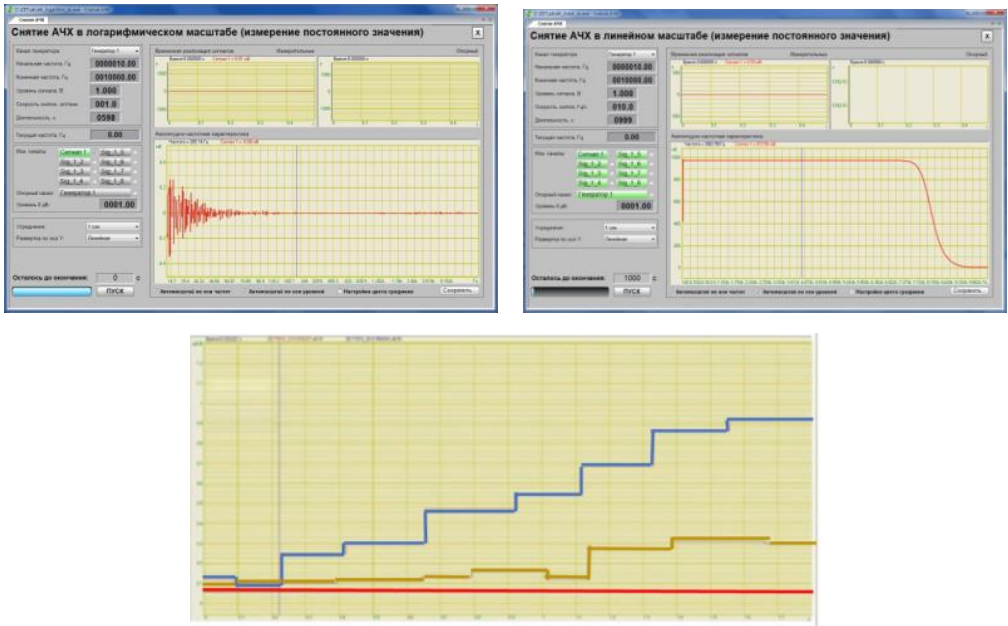

b) Results of research

Fig. 2. An example of research into the resource potential of the working body elements of the technological machine by tendometrication by the measuring system ZETLAB SENSOR. 
The discrepancy of the data is $8.9 \%$, which is within the limits of the acceptable $10 \%$. Comparisons of the received deformation relationships of the two measurements taken on different sections of the stand, allow us to conclude the validity of the studies conducted in the complex of automated design. It is also worth noting the errors in the work with tensomeasuring systems, due to possible interference with the frequency range of the current of $2.5 \mathrm{~Hz}$, and take into account the possible looseness of contact of the tensoresictor with the study surface.

The results of the research made it possible to create a universal apparatus of management of the resource potential of the technical systems of the technological machine for the entire life of the environmental management operations in conditions of high humidity and dusty external environment [15-17].

\section{Discussions}

In a practice, it is difficult to carry out such calculations, it is also economically unjustified to increase the frequency of service actions in a preventive manner, which logically leads to the conclusion that a digital module for monitoring the technical condition of the universal joint is included in the design of the digital module for monitoring the technical state of the universal joint, equipped with a calculation algorithm for evaluating parametric changes with an interface predictive notification of risk failure and planning of service actions by condition.

Technically, such concepts are feasible, since The control systems available in free circulation allow reprogramming control units for specific algorithms, the construction mechanism of which with the logical apparatus of mathematical and model support is presented in these studies. At the same time, the authors of the article propose to systematically expand the scope of innovative technologies, bringing them to the level of dual-use, as noted in previous scientific works [18-22].

It should be noted that such developments should be implemented by specialists in the field of technical operation of transport and transport-technological machines, and IT specialists should convert these algorithms into digital control modules.

For example, in the work of groups of authors was proposed the following:

- a tool for monitoring system failures in Industry 4.0 system is proposed, which focuses on system failures associated with resource localization [18];

- to use a fifth generation $(5 \mathrm{G})$ system that provides a coherent mapping between user equipment (UE) and remote radio point (RRH) and between RRHs and baseband processing unit $(\mathrm{BBU})$. The calculation results show that the proposed Bee-Ant-CRAN scheme for solving the problem of resource allocation: UE-rrh association and rrh-BBU mapping, (clustering) RRH-BBU reduces resource losses and significantly increases spectral efficiency [19].

Currently, with a high intensity of innovative proposals, the theory of "technological parasites" (Mario Coccia, Joshua Watts) is being developed, based on the idea of accelerating the evolution of parasitic-economic relations between technologies aimed at maintaining the competitive advantages of firms and nations. This theory offers a new direction for developing more complex concepts and theoretical foundations to explain technological and industrial changes in economic systems [20]. Frans Prenkert, et al. highlight the consistency of the analytical structure, recognizing the empirical variability of resource interfaces along with the consistent conceptualization of resources [21], which is in agreement with the works of leading Russian scientists V.A. Zorin and N.I. Baurova, who introduce the basic provisions of the theory of catastrophes into the analytical apparatus for assessing the resource of a technical system [22]. 


\section{Conclusions}

-It has been substantiated that, in accordance with the theory of probability, there can be only one failure in the unit at a time, even if several damages are diagnosed, then there is always a single cause, i.e. failure that caused a chain reaction of damage.

- The method of tribyometric control of the health of machines with the introduction of digital state accounting systems has been proposed.

-The construction of a diagnostic model by using the basic provisions of information theory allows representation the unit as a system containing the risk of uncertainty based on the sign of failure, which is distributed over all structural elements of the unit.

-An algorithm has been developed for the recognition rule for the area of displacement of uncertainty zones, the translation of which into a digital format with the introduction of a recognition criterion will provide for a defect identification (risk-failure).

-It is proposed to include in the design of technological machines a digital module for monitoring the technical state of the unit, including: a module for recognizing the elemental structure; a software shell with a calculation algorithm for evaluating parametric changes; interface predictive risk alert module; service control module by state.

- The results of the research made it possible to create a universal apparatus of management of the resource potential of the technical systems of the technological machine for the entire life of the environmental management operations in conditions of high humidity and dusty external environment.

\section{References}

1. Grib V.V., Zorin V.A., Zhukov R.V. Repair. Reconditioning. Modernization 6 19-22 (2016)

2. Duganova, E.V., Sevryugina, N.S., Kapyrin, P.D. IOP Conference Series: Materials Science and Engineering, 786(1), 012052, (2020)

3. Apatenko A.S. Analysis of processes and reasons for reducing the intensity of operation of technological machines Vestnik of Federal State Educational Establishment of Higher Professional Education "Moscow State Agroengineering University named after V P Goryachkin 3(59) 49-51 (2013)

4. Zorin V.A., Baurova N.I., Balovnev V.I., Grib V.V., Kosenko E.A. J. Russian Engineering Research 39 680-682 (2019)

5. Sevryugina N., Kapyrin P. MATEC Web of Conferences 17806017 (2018)

6. Kuznetsov A. P., Blau P., Koriath H-J., Richter M. J. Technological Machines and Production Engineering Procedia CIRP 46 340-343 (2016)

7. Hsin-Hui Chou, Judy Zolkiewski J. of Business Research 65(2) 188-195 (2012)

8. Niko Siltala, Eeva Järvenpää, Minna Lanz J. IFAC-Papers On Line 51(11) 102-107 (2018)

9. Chalaganidze S.I., Katsitadze J.B., Kutelia G.G. J. Annals of Agrarian Science 15(3) 329-331 (2017)

10. Valerie C.Y., Zhu Linyan Sun, Linhui Sun, Xiaohong Li J. Computers \& Industrial Engineering 58 (1) (2010)

11. Berg S., Wustmans M., Bröring S. Identifying first signal so emerging dominance in a technological in novation system: A novel approach based on patents Technological Forecasting and Social Change 146 706-722 (2019) 
12. Xiangxiang Sun, Xiaoliang Zhou, Zhangwang Chen, Yuping Yang Environmental efficiency of electric power industry, market segmentation and technological innovation: Empirical evidence from China Science of The Total Environment 706 135749 (2020)

13. Hung-Chun Huang, Hsin-Ning $\mathrm{Su}$ The innovative ful crums of technological interdisciplinarity: Ananalysis of technology fields in patents Technovation 84-85 5970 (2019)

14. Delu Wang, Xian Tong, Yadong Wang J. Resources Policy 66101593 (2020)

15. Sevryugina N.S., Apatenko A.S. Machinery and Equipment for Rural Area 7(265) 3538 (2019)

16. Sevryugina N.S., Apatenko A.S., Revyako S.I. IOP Conference Series: Materials Science and Engineering. The collection of conference materials. Voronezh State University of Engineering Technologies, 1001, 012983, (2020), doi:10.1088/1757899X/1001/1/012083

17. Apatenko A.S., Sevryugina N.S. IOP Conference Series: Materials Science and Engineering 786012037 (2019)

18. Bouziane Brik, Belgacem Bettayeb, M'hammed Sahnoun, Fabrice Duval Towards Predicting System Disruption in Industry 4.0: Machine Learning-Based Approach Procedia Computer Science 151 667-674 (2019)

19. Ado Adamou Abba Ari, Abdelhak Gueroui, Chafiq Titouna, Ousmane Thiare, Zibouda Aliouat, Resource allocation scheme for $5 G$ C-RAN: a Swarm Intelligence base dapproach Computer Networks 165106957 (2019)

20. Mario Coccia, Joshua Watts J. of Engineering and Technology Management 55101552 (2020)

21. Frans Prenkert, Nina Hasche, Gabriel Linton J. of Business Research 100 139-149 (2019)

22.Zorin V.A., Baurova N.I., Pegachkov A.A. Periodicals of Engineering and Natural Sciences 7(1) 287-293 (2019)

23. Zorin V., Baurova N. Assessment of means of mechanization of construction risks using catastrophe theory MATEC Web of Conferences 25103008 (2018) 